\title{
Keeping synapses quiet
}

\section{4}

both morphine administration and cocaine administration

... induce the same overall result in this pathway: an increase in the relative strength of excitatory drive to D1R MSNs versus D2R MSNs 5
Many drugs of abuse affect behaviour in part by inducing changes in synaptic transmission at excitatory synapses in the nucleus accumbens (NAc). However, paradoxically, morphine and cocaine produce similar behavioural effects (such as conditioned place preference and increased locomotor responses), but very different alterations at NAc synapses. Now, Graziane et al. find that morphine and cocaine both induce silent synapses in the NAc, which could account for the similar behavioural effects of these drugs.

Silent synapses contain postsynaptic NMDA receptors (NMDARs) but lack AMPA receptors (AMPARs). They are silent because, without AMPARs, the postsynaptic membrane does not depolarize in response to glutamate, meaning that the postsynaptic NMDARs - which open only under depolarizing conditions - remain inactivated.

Previous work has shown that in adult rats, 5 days of cocaine administration followed by 1 day of withdrawal increases the number of silent

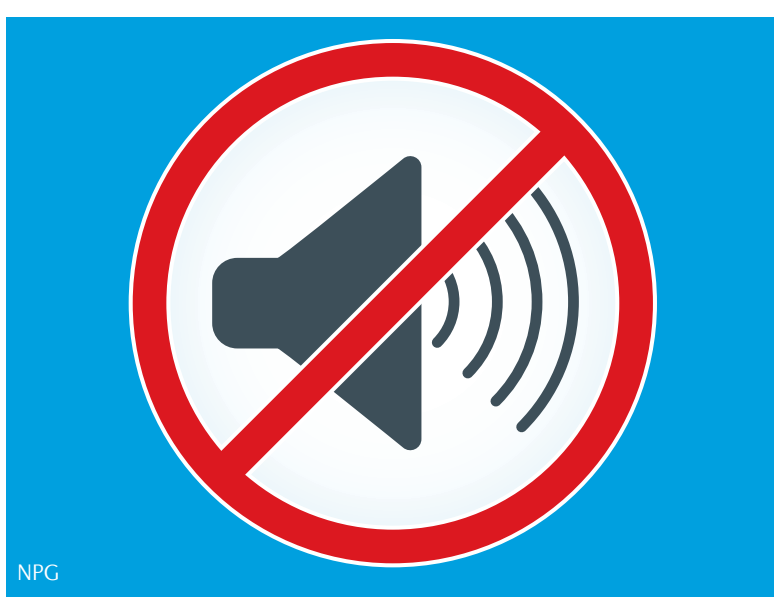

synapses onto dopaminergic medium spiny neurons (MSNs) in the NAc shell. Here, the authors repeated this dosing regimen with morphine and found that, similarly, there was an increase in the number of silent synapses onto NAc shell MSNs.

Silent synapses that result from repeated cocaine exposure typically include GluN2B-containing NMDARs, indicating that they are 'newly formed'. To investigate the mechanism by which morphine increases the number of silent synapses, Graziane et al. used a synthetic Tat-tagged peptide that prevents activity-mediated AMPAR internalization. Administration of the Tat-tagged peptide prevented the morphine-induced but not the cocaine-induced increase in the number of silent synapses, suggesting that the increase in silent synapses following morphine exposure is mediated by AMPAR internalization at existing synapses.

The authors also found that cocaine administration resulted in an increase in total spine density of MSNs in the NAc (with more long, thin spines - a morphology consistent with decreased stability), which was unaltered by administration of the Tat-tagged peptide. Morphine administration did not alter the total spine density but did induce an increase in the proportion of long, thin spines that was blocked by the Tat-tagged peptide, suggesting that AMPAR internalization could be involved in these changes. The authors used a mouse line in which dopamine D1 receptor (D1R)expressing MSNs were genetically tagged with tdTomato, and found that cocaine administration induced increases in silent synapses only onto these cells, whereas morphine increased silent synapses only onto D2R-expressing MSNs.

The authors reasoned that if the cocaine-induced spinogenesis and the increase in silent synapses become stabilized following drug withdrawal, the strength of the excitatory input to D1R-expressing MSNs would increase overall. Conversely, if the synapses that are weakened following repeated morphine administration were eliminated following drug withdrawal, the strength of excitatory input to D2R-expressing MSNs would be expected to decrease. The authors confirmed these predictions by simultaneously recording from D1R MSNs and D2R MSNs of mice after 3 weeks of withdrawal from either cocaine or morphine; following withdrawal from either drug, the ratio of excitatory input to D1R MSNs versus D2R MSNs was increased.

Therefore, both morphine administration and cocaine administration followed by drug withdrawal induce the same overall result in this pathway: an increase in the relative strength of excitatory drive to D1R MSNs versus D2R MSNs. Overall, these data suggest that although both cocaine and morphine induce similar behaviours, such as increased locomotion, they do so by inducing silent synapses through different mechanisms and in a cell-specific manner.

Sian Lewis

ORIGINAL ARTICLE Graziane, N. M. et al. Opposing mechanisms mediate morphine- and cocaine-induced generation of silent synapses. Nat. Neurosci. http://dx.doi.org/10.1038/nn.4313 (2016) 